\title{
Dimensionless parameters in symmetric double lap joints: an orthotropic solution for thermomechanical loading
}

\author{
Peter A. Gustafson; Arnaud Bizard $\rceil^{\dagger}$ and Anthony M. Waas ${ }^{\ddagger}$ \\ Department of Aerospace Engineering,University of Michigan,Ann Arbor, MI 48109, USA
}

\begin{abstract}
Two thermomechanical analytical models are proposed for orthotropic double lap joints, and are compared to a finite element model. The solutions, based on the principle of virtual work, differ in the complexity of the assumed stress field. The first solution is similar to Volkersen, with the addition of thermal effects. The second solution captures the peel stress as well as the traction free boundary condition at the adhesive edge. Relevant non-dimensional parameters are presented for geometric, material, and load quantities. A dimensionless load ratio is identified which dictates the shape of the stress distribution. This ratio can also be used to quickly determine the dominant loading mechanism. Dimensionless stress plots are presented for representative lap joints.
\end{abstract}

\section{Nomenclature}

$l \quad$ Lap length, $\mathrm{m}$

$t_{\kappa} \quad$ Material thicknesses of component $\kappa, \mathrm{m}$

$x \quad$ Lap coordinate measured from the left edge, $\mathrm{m}$

$y \quad$ Lap coordinate measured from the lower edge, $\mathrm{m}$

$\sigma_{\kappa 11(x)}$ Longitudinal stress in component $\kappa, \mathrm{Pa}$

$\sigma_{b 22(x)}$ Transverse stress in adhesive, $\mathrm{Pa}$

$\tau_{b 12(x)}$ Shear stress in the adhesive, $\mathrm{Pa}$

$E_{\kappa i i} \quad$ Orthotropic engineering moduli of component $\kappa, \mathrm{Pa}$

$E_{p[0 l]} \quad$ Young's moduli of the end posts, $\mathrm{Pa}$

$G_{b 12}$ Shear modulus of the adhesive, $\mathrm{Pa}$

$\alpha_{\kappa i i} \quad$ Orthotropic thermal expansion coefficients of component $\kappa, \mathrm{C}^{o-1}$

$\nu_{\kappa i j} \quad$ Poisson's ratios of component $\kappa$

$F \quad$ Mechanical load carried by an end post, $\mathrm{N}$

$P \quad$ Mechanical load applied to joint, per unit depth, $\mathrm{N} \mathrm{m}^{-1}$

$\Delta T \quad$ Temperature change from reference temperature, $\mathrm{C}^{o}$

$c_{0}, d_{0} \quad$ Coefficients of assumed stress distribution, $\mathrm{N}$

$c_{1}, d_{1} \quad$ Coefficients of assumed stress distribution, $\mathrm{N} \mathrm{m}^{-1}$

$\psi_{P} \quad$ Mechanical load parameter, $\mathrm{N} \mathrm{m}^{-4}$

$\phi_{P} \quad$ Mechanical load parameter, $\mathrm{N} \mathrm{m}^{-6}$

$\psi_{T} \quad$ Thermal load parameter, $\mathrm{N} \mathrm{m}^{-4}$

$\phi_{T} \quad$ Thermal load parameter, $\mathrm{N} \mathrm{m}^{-6}$

$\omega \quad$ System parameter, $\mathrm{m}^{-1}$

$\beta \quad$ System parameter, $\mathrm{m}^{-2}$

$\gamma \quad$ System parameter, $\mathrm{m}^{-4}$

$\bar{x} \quad$ Dimensionless coordinate $\frac{x}{l}$ measured from the left edge of the adhesive

$\bar{\omega} \quad$ Dimensionless system parameter

$\bar{\beta}, \bar{\gamma} \quad$ Dimensionless system parameters

*Graduate Student. Member AIAA. email: petegus@umich.edu

$\dagger$ Former Student

$\ddagger$ Professor. Associate Fellow, AIAA

Copyright (c) 2006 by Peter A. Gustafson. Published by the American Institute of Aeronautics and Astronautics, Inc. with permission. 
$\bar{\lambda}_{1}, \bar{\lambda}_{2} \quad$ Dimensionless system parameters

$\bar{\sigma}_{a 11(\bar{x})}$ Dimensionless longitudinal stress in the central adherend

$\bar{\sigma}_{c 11(\bar{x})}$ Dimensionless longitudinal stress in the outer adherend

$\bar{\tau}_{b 12(\bar{x})}$ Dimensionless shear stress

$\bar{\psi}_{P}, \bar{\phi}_{P}$ Dimensionless mechanical load parameters

$\bar{\psi}_{T}, \bar{\phi}_{T}$ Dimensionless thermal load parameter

$\bar{a}, \bar{b}, \bar{A}, \bar{B}, \bar{C}, \bar{D}$ Dimensionless integration constants

$\overline{\bar{a}}, \overline{\bar{b}}, \overline{\bar{A}}, \overline{\bar{B}}, \overline{\bar{C}}, \overline{\bar{D}}$ Dimensionless integration constants

\section{Subscripts}

[] the or operator, ie [12] is 1 or 2 (no sum)

$\kappa \kappa=[a b c]$ (no sum) representing central adherend (a), adhesive (b), and outer adherend $(c)$ respectively

ii $\quad i=[123]$ (no sum)

ij $\quad i, j=[123]$ where $i \neq j$ (no sum)

\section{Introduction}

$\mathrm{T}$ HE use of composite materials continues to increase in the aerospace industry, which places an ever increasing importance on the ability of designers to properly specify the performance of bonded structural joints. Due to specific strength, specific stiffness, and load distribution advantages, recent high profile aircraft and spacecraft have featured bonded joints. Epoxies and adhesives now becoming available have shown great promise to expand the temperature range over which structural fiber reinforced polymer composites are used. These materials provide an opportunity to replace specialized, non-structural thermal protection with integrated composite systems capable of carrying structural load over a range of temperature extremes. Consequently, temperature resistant composite structures and bonded joints will be used in increasing quantities. In addition to the harsh operating environments, the processing temperatures for these specialized epoxies and adhesives are also quite high. As a result, the materials carry a significant risk of adverse stress fields caused by differential thermal expansion, even at room temperature.

It has been claimed that approximately $70 \%$ of structural failures are initiated in joints, ${ }^{1}$ therefore great attention must be paid to proper design of joints. Engineers have long recognized that adhesively bonded joints reduce the stress concentrations associated with mechanical fasteners through a more even distribution of the transmitted load. As the adhesives available for bonding have improved, the use of bonded joints has enhanced or replaced the use of traditional mechanical fasteners in high performance aircraft. Confidence in such joints has grown with accumulated usage, as reflected in the use of bonded joints in aircraft including the Joint Strike Fighter, the Long Range Strike Aircraft, and Unmanned Aerial Vehicles. ${ }^{2}$ Additionally, the use of adhesively bonded composite joints has expanded into the automotive industry. ${ }^{\text {a }}$

Despite increased usage, the design of joints is often carried out in an ad-hoc fashion, relying heavily on physical testing and empirical models. If the role of temperature resistant composites is to expand, their use must be supported by an improved understanding of bonded joints. Further research is needed to expand modeling capability for bonded joints, as well as to determine the mechanical response of material systems. It is also important to transfer research level models into the product development environment.

In the present work, a brief review of the available literature for double lap joints is presented. Two thermomechanical models are proposed for the symmetric double lap joint, a joint commonly used in the aerospace industry. One model, referred to as the shear only model (SO), can be considered a thermomechanical extension to the work of Volkersen. ${ }^{3}$ A second model accounts for the traction free adhesive edges, and allows for calculation of the peel stresses. It will therefore be referred to as the shear-peel model (SP). Both models are then compared to a finite element (FE) model. The extension of the main findings of this work to a single lap joint is the subject of a separate investigation.

\section{A brief summary of double lap joint analytical models}

Many have offered analytical solutions to the bonded joint. First among them Volkersen, ${ }^{3}$ followed by the distinguished work by Goland and Reissner, ${ }^{4}$ both of which presented solutions to the single lap joint. In addition, their have been many authors (an incomplete list includes: Her, ${ }^{1}$ Hart-Smith, ${ }^{5}$ Peppiatt, ${ }^{6}$ Renton and Vinson, ${ }^{7}$ Allman, ${ }^{8}$

${ }^{\mathrm{a} C}$ Composites News International, Ashland Pliogrip Adhesive Bonds Roofs To Two BMW Sports Sedans, Jan 20, 2006 
and Yang $e t a l^{9}$ ) who have proposed models for single lap joints. (Additionally, summaries were provided by Benson $^{10}$ and Adams et al. ${ }^{11}$ ) Of greater interest to this work, several authors have addressed double lap joints including Her, ${ }^{1}$ Hart-Smith, ${ }^{12}$ Gilibert and Rigolot, ${ }^{13}$ and Sen and Jones, ${ }^{14} .{ }^{15}$ Though intended for single lap joints, the work of Volkersen could used for double lap joints with only slight modification.

There are three important phenomenon present in bonded lap joints which are often overlooked or ignored in the available analytical models. First, in an idealized lap joint, the edge of the adhesive is a traction free surface. Though this is captured in some models, it is not captured in the frequently cited work of Goland and Reissner, ${ }^{4}$ nor its descendents. For example, in the double lap solution of Chen and Nelson, ${ }^{16}$ the assumptions lead to a conclusion that the shear stress is maximized at the edge of the adhesive, instead of being zero. Several authors have presented double lap joint models which capture the traction free boundary condition, however the derived solutions are either numerical as in the case of Sen and Jones ${ }^{1415}$, or the governing equation is a differential equation of very high order as in the case of Whitney, ${ }^{17}$ where no intuition can be developed. It was noted by Benson ${ }^{10}$ that a minimum 4 th order differential equation is required to capture the traction free surfaces. The second solution of the present work has such a differential equation.

A second shortcoming that exists in many of the analytical and FE solutions is that most do not account for anisotropic material behavior. This is particularly a concern when they are used in reference to laminated composite materials, since transverse strength is often significantly lower than in-plane strength for a given laminate. ${ }^{12}$ Further, since high temperature curing cycles are frequently needed for temperature resistant materials, prudence dictates that anisotropic material behavior should be included in thermomechanical analytical models.

A third common oversight is also at the edge of the adhesive, this time at the material interface. In this location, there exists a geometric singularity which causes unbounded stress concentrations. ${ }^{18}$ Though non-linear material response might ensure that the stress remains finite, ${ }^{2}$ the peak stresses at the corners are very dependent on the specific geometry and material behavior. The possible effects are not considered here, since this aspect of the joint requires different modeling strategies that employ ideas rooted in fracture mechanics.

Temperature effects have been neglected in most analytical double lap joint models. The authors are aware of the work of Chen, ${ }^{16}$ Vinson, ${ }^{19}$ and Adams. ${ }^{20}$ The work of Vinson and Zumsteg ${ }^{19}$ is difficult to evaluate, in that it requires the solution of 18 simultaneous boundary conditions and can only be reasonably solved numerically. Qualitative assessments cannot be made since no plots of the predicted stresses were provided. In the of Adams $e t a l,{ }^{20}$ a numerical solution is required making it less useful as a design tool. Hart-Smith also considered thermal loading, though the solution did not capture the traction free boundary condition. ${ }^{12}$ Thermal loading cannot remain neglected since future materials will face high temperature loading during manufacturing (high temperature curing cycles) and in the operating environment (in hypersonic and re-entry vehicles).

\section{Analytically derived stress field in a double lap joint including thermal expansion}

\section{A. A model which assumes the adhesive carries shear stress only}

A schematic of a double lap joint is shown in Figure (1). In this work, a symmetric geometry is assumed, and two solutions will be proposed. The first solution will assume that the stress field varies only along the direction of loading. The adherends are assumed to carry normal stress only, and the adhesive is assumed to carry shear stress only. Due to symmetry, the bending moments present in the joint are assumed to be negligible. Under these assumptions, the stress field is a function of $x$ only. Thermal expansion is assumed to be linear with temperature. Plasticity, creep, and other non-linearities of the constituents are ignored, though it is likely that they could be significant. ${ }^{12}$

The central adherend is referred to as material $a$; an equilibrium element for the central adherend is pictured in Figure (2.1). Figure (2.2) represents the outer adherend, referred to as material $c$. In these two areas, $x$-equilibrium 


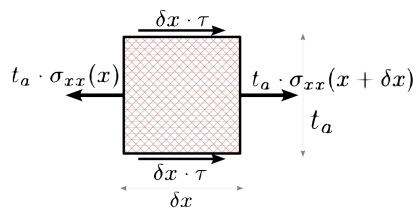

2.1: Equilibrium element of central adherend

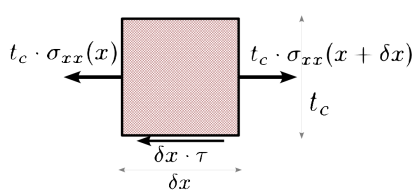

2.2: Equilibrium element of outer adherend

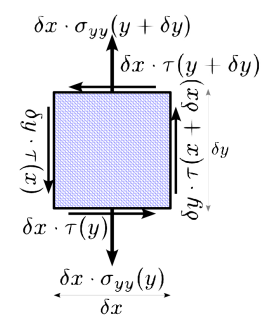

2.3: Equilibrium element of adhesive

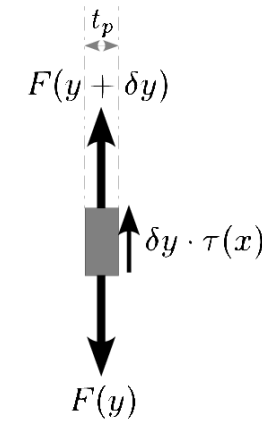

2.4: Equilibrium element of the left end post

requires the following:

$$
\begin{aligned}
& \frac{\partial \sigma_{a 11(x)}}{\partial x}=-\frac{2}{t_{a}} \tau_{b 12(x)}, \\
& \frac{\partial \sigma_{c 11(x)}}{\partial x}=-\frac{1}{t_{c}} \tau_{b 12(x)},
\end{aligned}
$$

where $x$ is measured from the left edge of the adhesive. Solving Eqs. (1) for $\tau_{b 12(x)}$ and equating leads to:

$$
t_{c} \frac{\partial \sigma_{c 11(x)}}{\partial x}=\frac{t_{a}}{2} \frac{\partial \sigma_{a 11(x)}}{\partial x} .
$$

The natural boundary conditions at the edge of adherend $a$ are:

$$
\begin{aligned}
\sigma_{a 11(0)} & =0, \\
\sigma_{a 11(l)} & =\frac{2 P}{t_{a}},
\end{aligned}
$$

which are the longitudinal normal stresses in the central adherend at the edges of the joint. Combining the above equations leads to the following relationship between stresses in the central and outer adherends:

$$
\sigma_{c 11(x)}=\frac{t_{a}}{2 t_{c}} \sigma_{a 11(x)}-\frac{P}{t_{c}} \text {. }
$$

Since the shear stress is assumed to be constant through the thickness of the adhesive, the shear stress in the adhesive is determined by Eqs. (1) and the solution to Eq. (4). Equations (1-4) can be solved using the principle of virtual forces, leading to a differential equation in the following form:

$$
\frac{\partial^{2} \sigma_{a 11(x)}}{\partial x^{2}}+\omega^{2} \sigma_{a 11(x)}+\psi_{T}+\psi_{P}=0 .
$$

Before stating the values of the system parameters $\omega^{2}, \psi_{T}$, and $\psi_{P}$, it is reasonable to non-dimensionalize the solution to Eq. (5), therefore the following substitutions are made:

$$
\begin{aligned}
\bar{\psi}_{T} & =\psi_{T} \frac{l^{2}}{E_{a 11}}, \\
\bar{\psi}_{P} & =\psi_{P} \frac{l^{2}}{E_{a 11}}, \\
\bar{x} & =\frac{x}{l}, \\
\bar{\omega} & =l \omega \\
\bar{\tau}_{b 12(\bar{x})} & =\frac{\tau_{b 12(l \bar{x})}}{E_{a 11}} \\
\bar{\sigma}_{a 11(\bar{x})} & =\frac{\sigma_{a 11(l \bar{x})}}{E_{a 11}} .
\end{aligned}
$$

$$
4 \text { of } 17
$$


In Eq. (6), the non-dimensional axial stress $\bar{\sigma}_{a 11(\bar{x})}$ could easily be confused for the axial strain $\varepsilon_{a 11}$, however this is not the case since the stress field is not uniaxial. In analytical models offered previously, the average shear stress $\tau_{b 12}^{a v e}$ has been chosen as the normalizing factor. However, since a thermal load without an externally applied mechanical load results in a zero average shear stress, the modulus of the central adherend $E_{a 11}$ is used for the normalization. Unfortunately, this choice looses the "stress concentration factor" associated with the average shear normalization, however it is necessary to avoid a singular result for thermal loads. Upon substitution, Eq. (5) becomes:

$$
\frac{\partial^{2} \bar{\sigma}_{a 11}}{\partial \bar{x}^{2}}+\bar{\omega}^{2} \bar{\sigma}_{a 11}+\bar{\psi}_{T}+\bar{\psi}_{P}=0
$$

which is a non-dimensional form of the governing equation. The parameters $\bar{\omega}^{2}, \bar{\psi}_{T}$ and $\bar{\psi}_{P}$ are given by:

$$
\begin{aligned}
& \bar{\omega}^{2}=\frac{2 G b_{12} l^{2}}{t_{b}}\left[\frac{\left(\nu_{c 13} \nu_{c 31}-1\right)}{E_{c 11} t_{c}}+\frac{2\left(\nu_{a 13} \nu_{a 31}-1\right)}{E_{a 11} t_{a}}\right], \\
& \bar{\psi}_{T}=\left[\frac{4 G b_{12} l^{2}\left(\alpha_{c 33} \nu_{c 31}-\alpha_{a 33} \nu_{a 31}+\alpha_{c 11}-\alpha_{a 11}\right)}{E_{a 11} t_{a} t_{b}}\right] \Delta T, \\
& \bar{\psi}_{P}=-\left[\frac{4 G b_{12} l^{2}\left(\nu_{c 13} \nu_{c 31}-1\right)}{E_{a 11} E_{c 11} t_{a} t_{b} t_{c}}\right] P .
\end{aligned}
$$

It is worth noting that Eq. (8) contains non-dimensional parameters for both thermal and mechanical loading. The thermal expansion of the adhesive is not a factor, since the adhesive is assumed to carry no longitudinal normal stress. In deriving the non-dimensional parameters, it has been assumed that the 2D geometry deforms in plane strain, and the material is orthotropic and aligned with the plane of deformation. A plane stress assumption could be substituted by setting all Poisson terms to zero.

The solution to Eq. (5) takes the form:

$$
\bar{\sigma}_{a 11(\bar{x})}=\bar{a} \sin (\bar{\omega} \bar{x})+\bar{b} \cos (\bar{\omega} \bar{x})-\frac{\bar{\psi}_{T}+\bar{\psi}_{P}}{\bar{\omega}^{2}},
$$

and possesses the following boundary conditions for longitudinal normal stress:

$$
\begin{aligned}
& \bar{\sigma}_{a 11}(0)=0 \\
& \bar{\sigma}_{a 11}(1)=\frac{2 P}{t_{a} E_{a 11}} .
\end{aligned}
$$

Application of the boundary conditions leads to the following values for the constants $\bar{a}, \bar{b}$ :

$$
\begin{aligned}
& \bar{a}=-\left[\frac{E_{c 11} t_{b} t_{c}}{2 G b_{12} l^{2} \sin \bar{\omega}\left(\nu_{c 13} \nu_{c 31}-1\right)}+\frac{\cos \bar{\omega}-1}{\bar{\omega}^{2} \sin \bar{\omega}}\right] \bar{\psi}_{P}-\frac{(\cos \bar{\omega}-1)}{\bar{\omega}^{2} \sin \bar{\omega}} \bar{\psi}_{\Delta T} \\
& \bar{b}=\frac{\bar{\psi}_{T}+\bar{\psi}_{P}}{\bar{\omega}^{2}}
\end{aligned}
$$

and the solution is completed.

The SO solution presented in this section minimizes solution complexity. As a result, it lacks certain desirable features. It does not offer a traction free adhesive edge, nor does it quantify the peel stress. Despite these shortcomings, the model is useful. It provides an analytical solution which includes consideration of thermal expansion. Also, important non-dimensional parameters have been identified in Eq. (8). These parameters dictate the joint stress distribution, and can be used as a first order analysis tool in the design of bonded double lap joints. Further, the SO solution provides the foundation for a formulation posed in the next section, the solution of which provides a zero traction at the adhesive edge.

\section{B. A model which assumes the adhesive carries shear and peel stress}

The second solution presented in this work is the SP extension to the above analysis. In this case, the adhesive is no longer confined to carry shear stress only. Instead, it is now assumed to carry shear and peel stresses, as shown in Figure (2.3). The adherends are assumed to be stiff, and carry only normal stresses as before. For convenience, a fictitious structural element referred to as an "end post" is located at the edge of the adhesive, and is assumed to be 
capable of transferring any shear stress at the edge towards the adherends. In making this assumption, the traction boundary condition is satisfied a priori. This modeling approach has been used in the design of shear panels, as described by Davies. ${ }^{21}$ The end post element, which is included in Figure (1), will be carried through the calculations and then eliminated at the end to restore the correct geometry.

The stress fields in the adherends are as described in the SO solution, with the exception of the peel stress in the adhesive layer. The $x$-equilibrium equations provided above still hold, however, $y$-equilibrium in the adhesive is now non-trivial.

Force equilibrium in the $y$ direction of the adhesive requires the following relation:

$$
\frac{\partial \sigma_{b 22(x)}}{\partial y}=-\frac{\partial \tau_{b 12(x)}}{\partial x}
$$

where $\sigma_{b 22(y)}$ is assumed to be a linear function of $y$ :

$$
\sigma_{b 22(y)}=c_{0}+c_{1} y
$$

By assumption, $\sigma_{b 22\left(t_{b}\right)}=0$, therefore:

$$
\sigma_{b 22(y)}=c_{0}\left(1-\frac{y}{t_{b}}\right)
$$

Combining Eq. (12) and Eq. (14) leads to:

$$
\sigma_{b 22(x, y)}=\frac{t_{a}}{2}\left(y-t_{b}\right) \frac{\partial^{2} \sigma_{a 11(x)}}{\partial x^{2}}
$$

Force equilibrium in the $y$ direction on the left end post requires the following relation:

$$
\frac{\partial F_{(y, x=0)}}{\partial y}=-\tau_{b 12(0)}
$$

where the force carried by the end post is also assumed to be a linear function of $y$ :

$$
F_{(y, x=0)}=d_{0}+d_{1} y
$$

Combining Eq. (17) with Eqs. (1) leads to:

$$
F_{(y, x=0)}=\frac{t_{a}}{2} \frac{\partial \sigma_{a 11(x=0)}}{\partial x} y+d_{0}
$$

Using similar arguments for the right end post, and applying the equilibrium requirement that the total end post force vanishes on each side, the end post governing equations are given by Eqs. (19).

$$
\begin{aligned}
& F_{(y, x=0)}=\frac{t_{a}}{2} \frac{\partial \sigma_{a 11(x=0)}}{\partial x}\left(y-\frac{t_{b}}{2}\right) \\
& F_{(y, x=l)}=-\frac{t_{a}}{2} \frac{\partial \sigma_{a 11(x=l)}}{\partial x}\left(y-\frac{t_{b}}{2}\right)
\end{aligned}
$$

With the equilibrium requirements now complete, application of the principal of virtual forces leads to a differential equation of the following form:

$$
\frac{\partial^{4} \sigma_{a 11(x)}}{\partial x^{4}}+\beta \frac{\partial^{2} \sigma_{a 11(x)}}{\partial x^{2}}+\gamma \sigma_{a 11(x)}+\phi_{T}+\phi_{P}=0 .
$$


Without explicit statement of the parameters, non-dimensionalizing substitutions can be made:

$$
\begin{aligned}
\bar{x} & =\frac{x}{l}, \\
\bar{\beta} & =l^{2} \beta, \\
\bar{\gamma} & =l^{4} \gamma, \\
\bar{\sigma}_{a 11(\bar{x})} & =\frac{\sigma_{a 11(l \bar{x})}}{E_{a 11}}, \\
\bar{\tau}_{b 12(\bar{x})} & =\frac{\tau_{b 12(l \bar{x})}}{E_{a 11}}, \\
\bar{\phi}_{T} & =\phi_{T} \frac{l^{4}}{E_{a 11}}, \\
\bar{\phi}_{P} & =\phi_{P} \frac{l^{4}}{E_{a 11}} .
\end{aligned}
$$

The solution of Eqs. $(1,15,19)$ as well as the non-dimensionalizing substitutions given in Eqs. (21) lead to the following differential equation for the normalized stress in the central adherend:

$$
\frac{\partial^{4} \bar{\sigma}_{a 11}}{\partial \bar{x}^{4}}+\bar{\beta} \frac{\partial^{2} \bar{\sigma}_{a 11}}{\partial \bar{x}^{2}}+\bar{\gamma} \bar{\sigma}_{a 11}+\bar{\phi}_{T}+\bar{\phi}_{P}=0
$$

where the dimensionless system parameters are given by:

$$
\begin{aligned}
\bar{\beta} & =\frac{3 E_{b 22} l^{2}}{2 G b_{12} t_{b}^{2}\left(\nu_{b 23} \nu_{b 32}-1\right)}, \\
\bar{\gamma} & =\frac{3 E_{b 22} l^{4}\left(E_{a 11} t_{a} \nu_{c 13} \nu_{c 31}+2 E_{c 11} t_{c} \nu_{a 13} \nu_{a 31}-2 E_{c 11} t_{c}-E_{a 11} t_{a}\right)}{E_{a 11} E_{c 11} t_{a} t_{b}^{3} t_{c}\left(\nu_{b 23} \nu_{b 32}-1\right)}, \\
\bar{\phi}_{T} & =\left[\frac{6 E_{b 22} l^{4}\left(\alpha_{c 33} \nu_{c 31}-\alpha_{a 33} \nu_{a 31}+\alpha_{c 11}-\alpha_{a 11}\right)}{E_{a 11} t_{a} t_{b}^{3}\left(\nu_{b 23} \nu_{b 32}-1\right)}\right] \Delta T \\
\bar{\phi}_{P} & =-\left[\frac{6 E_{b 22} l^{4}\left(\nu_{c 13} \nu_{c 31}-1\right)}{E_{a 11} E_{c 11} t_{a} t_{b}^{3} t_{c}\left(\nu_{b 23} \nu_{b 32}-1\right)}\right] P .
\end{aligned}
$$

The solution takes the following form:

$$
\bar{\sigma}_{a 11}(\bar{x})=\bar{A} e^{\bar{\lambda}_{1} \bar{x}}+\bar{B} e^{-\bar{\lambda}_{1} \bar{x}}+\bar{C} e^{\bar{\lambda}_{2} \bar{x}}+\bar{D} e^{-\bar{\lambda}_{2} \bar{x}}-\frac{\bar{\phi}_{T}}{\gamma}-\frac{\bar{\phi}_{P}}{\gamma} .
$$

The bi-quadratic Eq. (24) has two dimensionless system parameters $\bar{\lambda}_{1}$ and $\bar{\lambda}_{2}$ given by Eq. (25) and presented in terms of the orthotropic material properties in Appendix (A).

$$
\bar{\lambda}_{[12]}^{2}=\frac{-\bar{\beta} \pm \sqrt{\bar{\beta}^{2}-4 \bar{\gamma}}}{2} .
$$

The integration constants $\bar{A}, \bar{B}, \bar{C}$, and $\bar{D}$ from Eq. (24) are determined by application of the boundary conditions, presented in full form in Appendix (A) as Eqs. (38) and in reduced form in Eqs. (26). These boundary conditions represent axial normal stress and shear stress at the ends of the central adherend. The reduced form is achieved by allowing the end posts to approach zero thickness (taking the limit as $t_{p} \rightarrow 0$ ). This procedure has the direct effect of forcing the shear stress at the post locations to zero, which results in a traction free surface at the adhesive edge.

$$
\begin{aligned}
\bar{D}+\bar{C}+\bar{B}+\bar{A}-\frac{\bar{\phi}_{T}+\bar{\phi}_{P}}{\bar{\gamma}} & =0 \\
e^{\bar{\lambda}_{4}} \bar{D}+e^{\bar{\lambda}_{2}} \bar{C}+e^{\bar{\lambda}_{2}} \bar{B}+e^{\bar{\lambda}_{1}} \bar{A}-\frac{\bar{\phi}_{T}+\bar{\phi}_{P}}{\bar{\gamma}}-\frac{2 P}{E_{a 11} t_{a}} & =0 \\
\bar{\lambda}_{4} \bar{D}+\bar{\lambda}_{2} \bar{C}+\bar{\lambda}_{2} \bar{B}+\bar{\lambda}_{1} \bar{A} & =0 \\
\bar{\lambda}_{4} e^{\bar{\lambda}_{4}} \bar{D}+\bar{\lambda}_{2} e^{\bar{\lambda}_{2}} \bar{C}+\bar{\lambda}_{2} e^{\bar{\lambda}_{2}} \bar{B}+\bar{\lambda}_{1} e^{\bar{\lambda}_{1}} \bar{A} & =0
\end{aligned}
$$


The solution of Eqs. (26) for $\bar{A}, \bar{B}, \bar{C}$, and $\bar{D}$ requires lengthy combinations of the system parameters. They are presented in a compact form in Eqs. (27), where certain repeating values have been represented as a series of multipliers $\mu$. The values of these $\mu$ parameters are presented in Appendix (B). With the presentation of Eqs. (27), the SP solution is now completed.

$$
\begin{aligned}
& \bar{A}=\frac{\mu_{A_{T}} \bar{\phi}_{\Delta T}+\left(\mu_{A_{T}}+\mu_{2} \mu_{3} \mu_{A_{P}}\right) \bar{\phi}_{P}}{\mu_{1}} \\
& \bar{B}=\frac{\mu_{B_{T}} \bar{\phi}_{\Delta T}+\left(\mu_{B_{T}}+\mu_{2} \mu_{3} \mu_{B_{P}}\right) \bar{\phi}_{P}}{\mu_{1}} \\
& \bar{C}=\frac{\mu_{C_{T}} \bar{\phi}_{\Delta T}+\left(\mu_{C_{T}}+\mu_{2} \mu_{3} \mu_{C_{P}}\right) \bar{\phi}_{P}}{\mu_{1}} \\
& \bar{D}=\frac{\mu_{D_{T}} \bar{\phi}_{\Delta T}+\left(\mu_{D_{T}}+\mu_{2} \mu_{3} \mu_{D_{P}}\right) \bar{\phi}_{P}}{\mu_{1}}
\end{aligned}
$$

The SP solution presented above overcomes some of the effects previously ignored by other analytical solutions in the literature. Most significantly, it is a quantitative thermomechanical solution which also ensures that the shear stress at the traction free edge is zero. It does so with the minimal required complexity of a fourth order governing differential equation.

The analysis is an elastic solution, and as a result neglects the effect of adherent and adherend plasticity, if any, on the joint. However, this effect was addressed analytically in Hart-Smith. ${ }^{12}$ Despite its potential importance, the inclusion of plasticity effects are best treated through a numerical solution.

\section{A dimensionless ratio of thermal and mechanical loading factors}

Using the non-dimensional loading parameters defined in Eqs. (8) and (23), a dimensionless load ratio $\left(\bar{\phi}_{a R}\right)$ and total load $\left(\bar{\phi}_{\text {total }}\right)$ can be defined.

$$
\begin{aligned}
\bar{\phi}_{a R} & =\frac{\bar{\phi}_{T}}{\bar{\phi}_{P}}=-\frac{E_{c 11} t_{c}\left(\alpha_{c 33} \nu_{c 31}-\alpha_{a 33} \nu_{a 31}+\alpha_{c 11}-\alpha_{a 11}\right) \Delta T}{\left(\nu_{c 13} \nu_{c 31}-1\right) P} \\
\bar{\phi}_{\text {total }} & =\bar{\phi}_{P}+\bar{\phi}_{T}
\end{aligned}
$$

The ratio $\bar{\phi}_{a R}$ is a measure of the relative importance of allowable thermal and mechanical loads. The importance of the load ratio $\bar{\phi}_{[a b] R}$ must no be underestimated. When $\left|\bar{\phi}_{[a b] R}\right| \ll 1$, mechanical stress dominates the stress field in the adherend. Conversely, when $\left|\bar{\phi}_{[a b] R}\right| \gg 1$, the thermally induced stress field is dominant. Finally, when $\left|\bar{\phi}_{[a b] R}\right| \approx 1$, thermal and mechanical loads are both significant to the total stress field. Using $\bar{\phi}_{a R}$ as a guide, it is easy to see how common joints such as Aluminum to carbon fiber reinforced polymer matrix composites can be dominated by thermal loading when a large $\Delta T$ is present. It is significant that the dimensionless load ratio is the same whether the SO or the SP is used to derive it, as it is therefore independent of the adhesive stress field assumption.

The stress field that leads to the dimensionless number given in Eqs. (28) is based on the stress in the central adherend $\bar{\sigma}_{a 11}(\bar{x})$. Using Eq. (4) and similarly collecting terms into dimensionless loads, a conjugate dimensionless load ratio can be written for the stress field in the outer adherend $\bar{\sigma}_{c 11}(\bar{x})$;

$$
\bar{\phi}_{c R}=\frac{E_{a 11} t_{a}\left(\alpha_{c 33} \nu_{c 31}-\alpha_{a 33} \nu_{a 31}+\alpha_{c 11}-\alpha_{a 11}\right) \Delta T}{2\left(\nu_{a 13} \nu_{a 31}-1\right) P} .
$$

Examining Eqs. (28) and (29), it is apparent that the dimensionless load ratio in one adherend depends largely on the stiffness of the other adherend.

With the dimensionless load ratio in mind, a load-based normalization can be defined by rewriting the axial stress as:

$$
\overline{\bar{\sigma}}_{a 11}=\frac{\bar{\sigma}_{a 11}}{\bar{\phi}_{\text {total }}},
$$

or, more intuitively:

$$
\bar{\sigma}_{a 11(\bar{x})}=\overline{\bar{\sigma}}_{a 11\left(\overline{\bar{\phi}}_{P}, \bar{x}\right)} \cdot \bar{\phi}_{t o t a l}
$$


This second normalization can be propagated throughout the solution so that the SO and SP solutions are written as:

$$
\begin{aligned}
& \overline{\bar{\sigma}}_{a 11(\bar{x})}=\overline{\bar{a}} \sin (\bar{\omega} \bar{x})+\overline{\bar{b}} \cos (\bar{\omega} \bar{x})-\frac{1}{\bar{\omega}^{2}} \\
& \overline{\bar{\sigma}}_{a 11(\bar{x})}=\overline{\bar{A}} e^{\bar{\lambda}_{1} \bar{x}}+\overline{\bar{B}} e^{-\bar{\lambda}_{1} \bar{x}}+\overline{\bar{C}} e^{\bar{\lambda}_{2} \bar{x}}+\overline{\bar{D}} e^{-\bar{\lambda}_{2} \bar{x}}-\frac{1}{\gamma} .
\end{aligned}
$$

Doing so requires that the boundary conditions be rewritten as:

$$
\begin{aligned}
\overline{\bar{\sigma}}_{a 11}(0) & =0, \\
\overline{\bar{\sigma}}_{a 11}(1)-\frac{2 P}{t_{a} E_{a 11} \bar{\phi}_{\text {total }}} & =0,
\end{aligned}
$$

for the SO solution, and for the SP solution as:

$$
\begin{aligned}
& \overline{\bar{D}}+\overline{\bar{C}}+\overline{\bar{B}}+\overline{\bar{A}}-\frac{1}{\bar{\gamma}}=0 \\
& e^{\bar{\lambda}_{4}} \overline{\bar{D}}+e^{\bar{\lambda}_{2}} \overline{\bar{C}}+e^{\bar{\lambda}_{2}} \overline{\bar{B}}+e^{\bar{\lambda}_{1}} \overline{\bar{A}}-\frac{1}{\bar{\gamma}}-\frac{2 P}{E_{a 11} t_{a} \bar{\phi}_{\text {total }}}=0 \\
& \bar{\lambda}_{4} \overline{\bar{D}}+\bar{\lambda}_{2} \overline{\bar{C}}+\bar{\lambda}_{2} \overline{\bar{B}}+\bar{\lambda}_{1} \overline{\bar{A}}=0 \\
& \bar{\lambda}_{4} e^{\bar{\lambda}_{4}} \overline{\bar{D}}+\bar{\lambda}_{2} e^{\bar{\lambda}_{2}} \overline{\bar{C}}+\bar{\lambda}_{2} e^{\bar{\lambda}_{2}} \overline{\bar{B}}+\bar{\lambda}_{1} e^{\bar{\lambda}_{1}} \overline{\bar{A}}=0 .
\end{aligned}
$$

Using the load ratio $\bar{\phi}_{a R}$, we can split the integration constants into linear equations of the mechanical fraction of the load. Defining the mechanical load fraction as:

$$
\overline{\bar{\phi}}_{P}=\frac{\bar{\phi}_{P}}{\bar{\phi}_{\text {total }}}=\left(1+\bar{\phi}_{a R}\right)^{-1},
$$

the constants $\overline{\bar{a}}$ and $\overline{\bar{b}}$ from Eqs. (9) for a load normalized solution can be written as:

$$
\begin{aligned}
& \overline{\bar{a}}=-\frac{E_{c 11} t_{b} t_{c}}{2 G b_{12} l^{2} \sin \bar{\omega}\left(\nu_{c 13} \nu_{c 31}-1\right)} \overline{\bar{\phi}}_{P}-\frac{\cos \bar{\omega}-1}{\bar{\omega}^{2} \sin \bar{\omega}}, \\
& \overline{\bar{b}}=\frac{1}{\bar{\omega}^{2}} .
\end{aligned}
$$

Similarly, the $\overline{\bar{A}}, \overline{\bar{B}}, \overline{\bar{C}}$, and $\overline{\bar{D}}$ integration constants can be written as:

$$
\begin{aligned}
& \overline{\bar{A}}=\frac{\mu_{3} \mu_{A_{P}}}{\mu_{1} \mu_{2}} \overline{\bar{\phi}}_{P}+\frac{\mu_{A_{T}}}{\mu_{1}}, \\
& \overline{\bar{B}}=\frac{\mu_{3} \mu_{B_{P}}}{\mu_{1} \mu_{2}} \overline{\bar{\phi}}_{P}+\frac{\mu_{B_{T}}}{\mu_{1}}, \\
& \overline{\bar{C}}=\frac{\mu_{3} \mu_{C_{P}}}{\mu_{1} \mu_{2}} \overline{\bar{\phi}}_{P}+\frac{\mu_{C_{T}}}{\mu_{1}}, \\
& \overline{\bar{D}}=\frac{\mu_{3} \mu_{D_{P}}}{\mu_{1} \mu_{2}} \overline{\bar{\phi}}_{P}+\frac{\mu_{D_{T}}}{\mu_{1}},
\end{aligned}
$$

where the $\mu$ parameters are given in Appendix (B). In this form, it becomes apparent that the integration constants $\overline{\bar{a}}$, $\overline{\bar{b}}, \overline{\bar{A}}, \overline{\bar{B}}, \overline{\bar{C}}, \bar{D}$ (and by extension $\bar{a}, \bar{b}, \bar{A}, \bar{B}, \bar{C}, \bar{D}$ ) govern the stress distribution via the thermal and mechanical load ratio, $\bar{\phi}_{a R}$, enhancing its relevance to the study of thermomechanical loading of lap joints.

The forms presented in Eqs. (36) and (37) will allow for an iterative version of the SO or SP solution to be applied using numerical methods, when the mechanical load is dependent on the thermal load. For example, this would allow for solution of displacement constrained thermal mechanical problems.

\section{A finite element model for the symmetric double lap joint}

To establish confidence in the SO and SP models proposed in Section (III), it is appropriate to compare the solution with a FE model. Therefore, a 2D FE model has been generated for the ASTM International double lap joint. ${ }^{22}$ An 


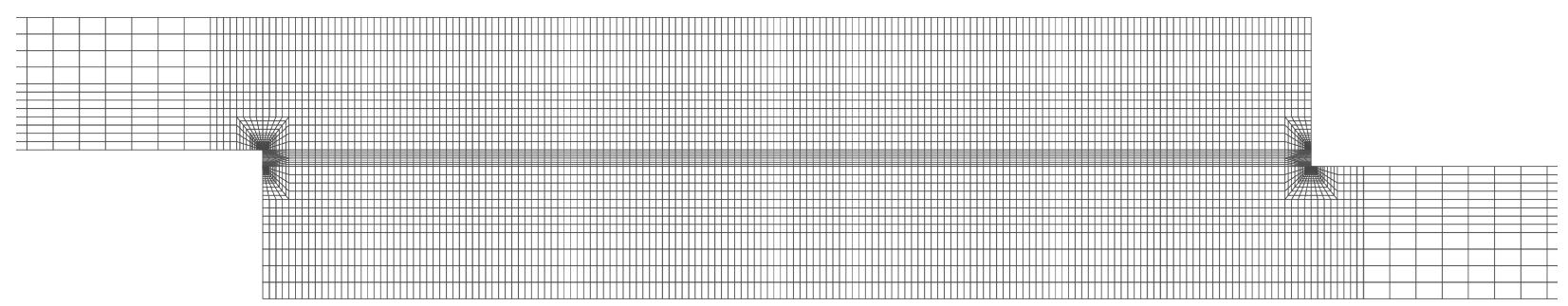

Figure 2: The FE mesh

overview of the mesh is shown in Figure (2), and the assumed geometries are given in Table (1(a)). The solver used is CalculiX, ${ }^{23}$ and the mesh consists entirely of quadratic plain strain elements (CPE8). Half of the joint is modeled due to symmetry. The stress concentrations at the material interfaces are generally resolved in the vicinity of the corner with a very fine mesh, though the geometric singularity is not (and cannot) be resolved with the FE technique used here. Loading is specified as listed in Table (1(b)), where the mechanical load is applied far away from the lap joint and the thermal load is applied to all nodes. Displacement symmetry constraints are enforced along the mid-plane of the central adherend. Non-linear geometric stiffness is assumed.

Table 1: Geometric and loading assumptions for model comparison

(a) ASTM double lap joint geometric features (mm).

\begin{tabular}{ccc} 
Component & Thickness & Length \\
\hline Outer Adherend & 1.6 & 76.2 \\
Adhesive & 0.2 or 1.0 & 12.7 \\
Central Adherend & 3.2 & 76.2
\end{tabular}

(b) Assumed loading.

\begin{tabular}{cc} 
Load Type & Value \\
\hline$P\left(\mathrm{~N} \cdot \mathrm{mm}^{-1}\right)$ & 10 \\
$\Delta T\left({ }^{\circ} \mathrm{C}\right)$ & 10
\end{tabular}

Aluminum ( $\mathrm{Al}$ ) is used as the central adherend in all models; the outer adherends are Al, Titanium (Ti), and AS4/3501-6 (AS4). ${ }^{24}$ For simplicity, the adhesive properties are assumed to be isotropic, and are estimated base on Cytec FM300 adhesive. The assumed material properties are summarized in Table (2). Reported stresses from the FE model are taken from the mid plane of the adhesive for shear stress. Peel stress is reported at the central adherend material interface in the FE and SP models.

Table 2: Assumed material properties in FE, SO, and SP solutions (moduli in GPa, expansion coeffs. in $\mu \epsilon^{\circ} \mathrm{C}^{-1}$ )

\begin{tabular}{ccccccccccccc} 
Material & $E_{11}$ & $E_{22}$ & $E_{33}$ & $G_{12}$ & $G_{13}$ & $G_{23}$ & $\nu_{12}$ & $\nu_{13}$ & $\nu_{23}$ & $\alpha_{11}$ & $\alpha_{22}$ & $\alpha_{33}$ \\
\hline Aluminum & 70 & 70 & 70 & 26.3 & 26.3 & 26.3 & 0.33 & 0.33 & 0.33 & 23 & 23 & 23 \\
Titanium & 110 & 110 & 110 & 41.4 & 41.4 & 41.4 & 0.33 & 0.33 & 0.33 & 9 & 9 & 9 \\
AS4/3501-6 $\left(0^{\circ}\right)$ & 148 & 10.6 & 10.6 & 5.61 & 5.61 & 3.17 & 0.30 & 0.30 & 0.59 & -0.8 & 29 & 29 \\
AS4/3501-6 $\left(90^{\circ}\right)$ & 10.6 & 148 & 10.6 & 5.61 & 3.17 & 5.61 & 0.30 & 0.59 & 0.30 & 29 & -0.8 & 29 \\
FM300 & 1.98 & 1.98 & 1.98 & 0.71 & 0.71 & 0.71 & 0.40 & 0.40 & 0.40 & 20 & 20 & 20
\end{tabular}

\section{Comparison of FE and analytical model results for ASTM lap specimens}

Figures (3-7) show the stress response predicted by the SO, SP, and FE models due thermal and mechanical loads applied to several joints. Examining the Al-Al joint shown in Figures (3.1,3.4), it is found that all three models result in approximately zero stress due to thermal loading. This intuitive result confirms the assumption that the differential expansion between the adhesive and adherends is negligible to the total stress field.

Model predictions for an Al-Al joint with applied mechanical load are shown in Figures (3.2,3.5). The first of these 


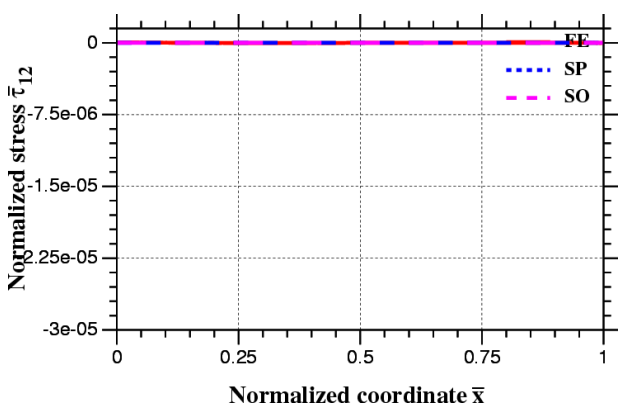

3.1: $\bar{\tau}_{b 12}$ due to $\bar{\phi}_{T}$
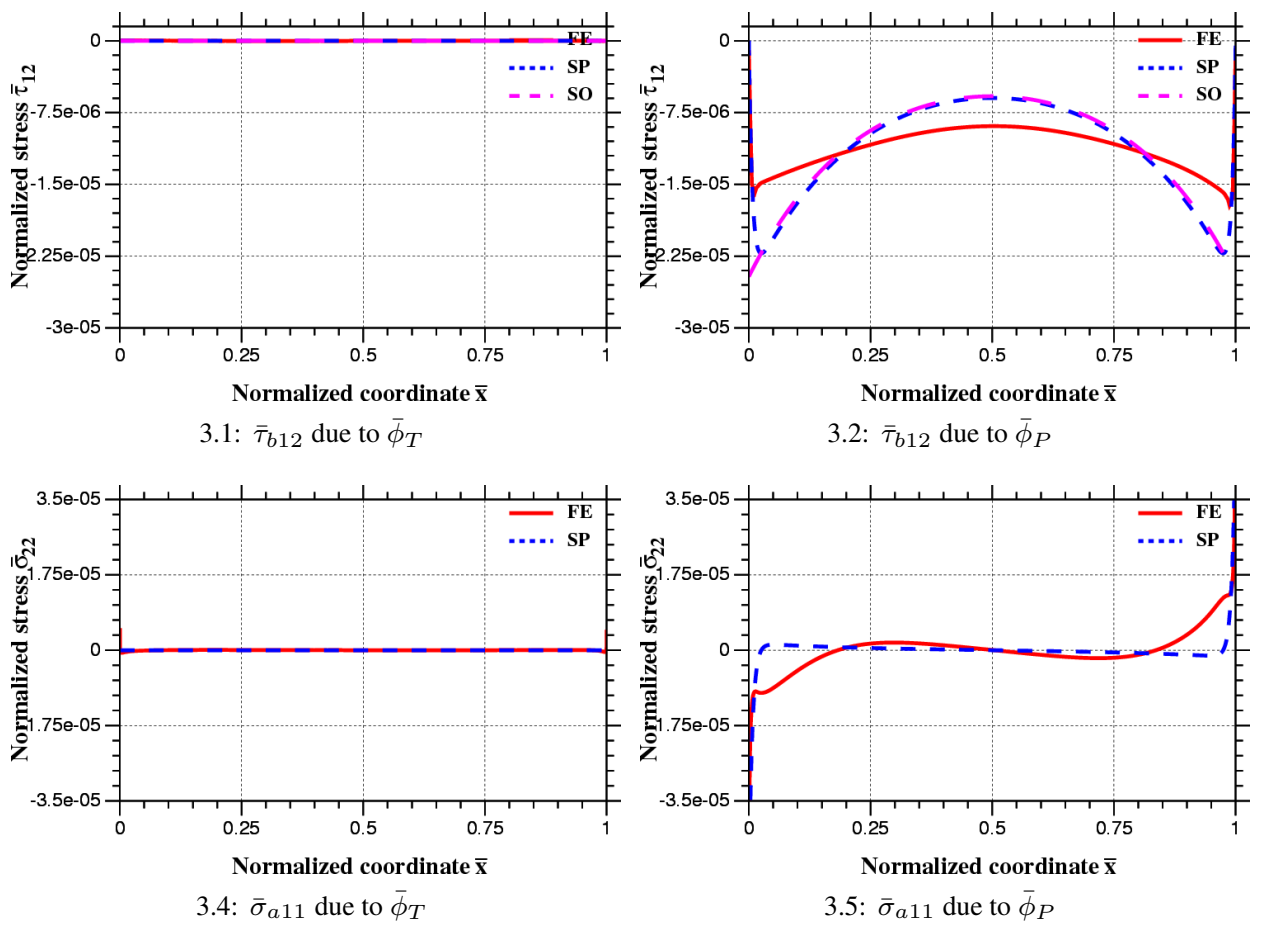

Normalized coordinate $\bar{x}$

3.2: $\bar{\tau}_{b 12}$ due to $\bar{\phi}_{P}$

Figure 3: FE, SP, and SO models of Al-Al double lap joint with $0.2 \mathrm{~mm}$ FM300 adhesive. $\bar{\phi}_{a R}=0$

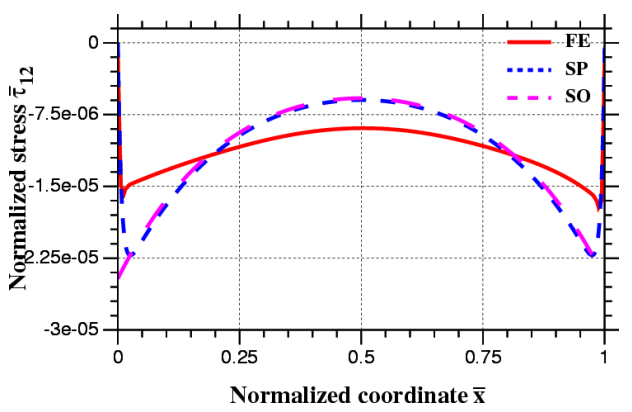

3.3: $\bar{\tau}_{b 12}$ due to $\bar{\phi}_{P}+\bar{\phi}_{T}$

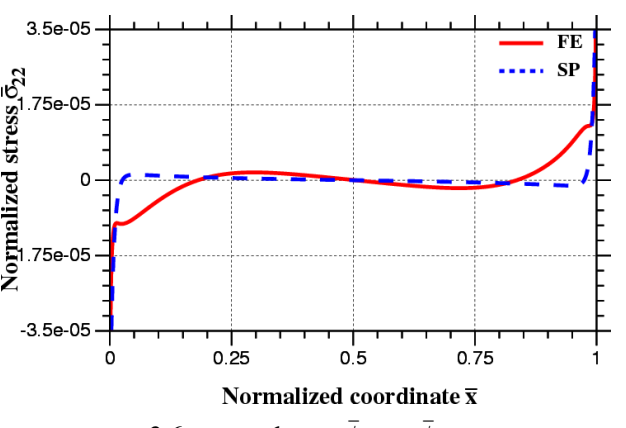

3.6: $\bar{\sigma}_{a 11}$ due to $\bar{\phi}_{P}+\bar{\phi}_{T}$

two figures shows the normalized shear stress $\bar{\tau}_{b 12}$ in the joint. The agreement between the FE, SO, and SP models is generally good, though the SO and SP models over predict the shear stress near the edges, in comparison to the FE solution. Total shear (the area under the $\bar{x}$ - $\bar{\tau}_{b 12}$ curves) is preserved, because the SO and SP solutions under predict the stress in the middle of the joint relative to the FE model. The traction free boundary condition is captured by the FE and SP solutions only, as expected.

The plots in Figures (3.5) show the peel stress due to mechanical load, as predicted by FE and SP solutions. It is apparent that differences exist in predicted peel stress, particularly in the areas at a modest distance inside the edges of the joint. However, at or near the edges of the joint, the agreement is excellent. This strong correlation between the two solutions at the edge locations is important, since peel stress is often a cause of failure in lap joints. Similarly, Figures $(3.3,3.6)$ show the predicted stress state due to a mixed loading condition, where both thermal and mechanical loads are applied. In the case of the Al-Al joint, it is clear that mechanical loading dominates the stress state. This result is fully expected, since the loading ratio, $\bar{\phi}_{a R}$, is zero.

The FE, SO, and SP model predictions for Al-Ti lap joints are shown in Figures (4-5). There are several important conclusions that can be drawn by examining these models. First, Figures (4.1) and (5.1) show agreement in trend between the FE model and the SO and SP models when thermal loading is applied. The predicted shear stress is zero in the middle of the joint, which is required when there is no mechanical load. Also, the SO and SP solutions for mechanical load in Figures (4.2,4.4) and Figures (5.2,5.4) have similar agreement to the Al-Al joint.

Mixed loading for Al-Ti joints is shown in Figures $(4.3,4.6)$ and $(5.3,5.6)$. These figures show that the SO and SP solutions compare well with the FE solution over the majority of the joint when the loading is thermal and mechanical. However, mixed loading results must be interpreted carefully, since the two loading types can produce shears of opposite sign in the same location. When this is the case, the relative magnitudes of the shear and thermal loads play a critical role, particularly at the ends of the joint. At the left edge of the lap zone in Figures (5.1-5.3), it shown that the thermally induced shear stress predicted by the SO and SP models is stronger than the mechanically induced shear stress. The total shear stress due to mixed loading is a combination of the two sources. Since the peel stress in the SP model is calculated using the derivative of the total shear stress, this leads to a significant difference in peel stress predictions between FE and SP models at this location. However, since the shear stresses from the thermal and mechanical loads act to against each other, it is unlikely that this is a critical location. Therefore, solution accuracy is much more important at the right edge, where the predicted shear and peel stresses are in good agreement with the FE 
model.

As in the Al-Al comparison, both SO and SP models tend to over predict the shear stress in the Al-TI joint, and the SP solution reasonably predicts the peel stress near the edges of the joint. Finally, comparing Figures (4.1-4.6) and (5.1-5.6), it is shown that both the SO and SP models correctly predict the stress field trend as the thickness of the adhesive is increased.

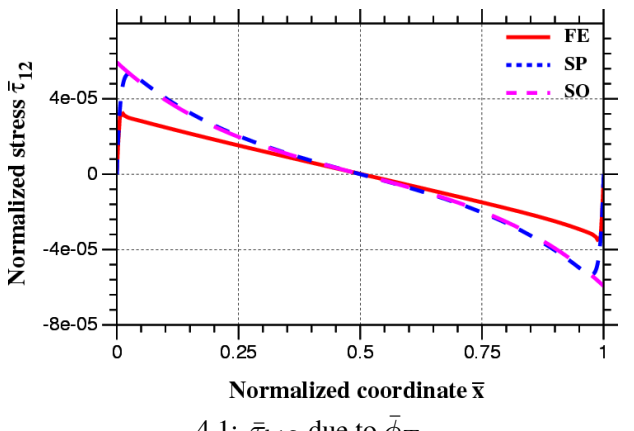

4.1: $\bar{\tau}_{b 12}$ due to $\bar{\phi}_{T}$

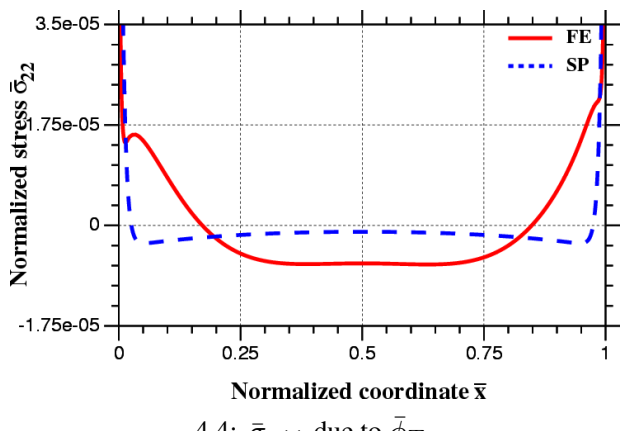

4.4: $\bar{\sigma}_{a 11}$ due to $\bar{\phi}_{T}$

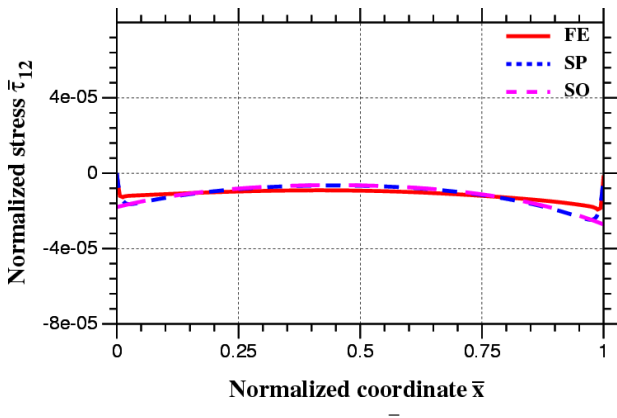

4.2: $\bar{\tau}_{b 12}$ due to $\bar{\phi}_{P}$

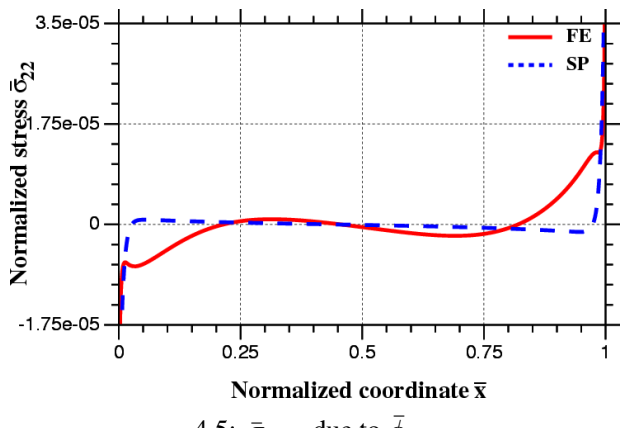

4.5: $\bar{\sigma}_{a 11}$ due to $\bar{\phi}_{P}$

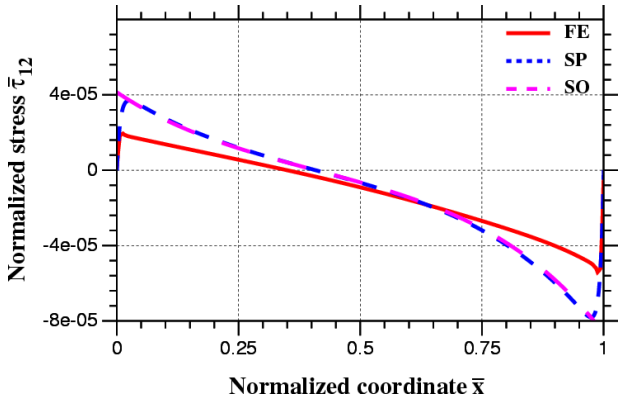

4.3: $\bar{\tau}_{b 12}$ due to $\bar{\phi}_{P}+\bar{\phi}_{T}$

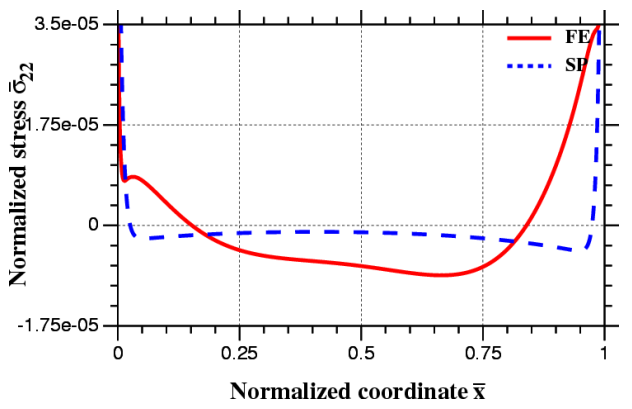

4.6: $\bar{\sigma}_{a 11}$ due to $\bar{\phi}_{P}+\bar{\phi}_{T}$

Figure 4: FE, SP, and SO models of Al-Ti double lap joint with $0.2 \mathrm{~mm} \mathrm{FM300} \mathrm{adhesive.} \bar{\phi}_{a R}=-3.68$

The stress predictions for the Al-AS4 joints are shown in Figures (6-7), where uniaxial fiber alignment for the orthotropic AS4 is aligned with the $x$ axis in Figures (6), and with the $z$ axis in (7). Though the latter is an unlikely joint arrangement, it is a useful exercise to examine the orthotropic nature of the SO and SP solutions. It is immediately apparent in $(6.1,6.4)$ and $(7.1,7.4)$ that differences in the orthotropic expansion coefficients have a significant effect. The sign of the stress changes upon a $90^{\circ}$ orientation change, and the magnitude of the stress is significantly lower as the fibers are aligned perpendicular to the cross section of the joint. This result makes sense, since the material is much more compliant when loaded in the $90^{\circ}$ orientation.

Upon examining all predicted stress results in Figures (3-7), the effect of the thermomechanical load ratio $\bar{\phi}_{a R}$ is apparent. The Al-Al joint, at $\left|\bar{\phi}_{a R}\right|=0$, is dominated by mechanical load. Both Al-Ti joints, at $\left|\bar{\phi}_{a R}\right|=3.68$, have significant contributions from both thermal and mechanical load. Comparing Al-AS4 $\left(0^{\circ}\right)$ and $\left(90^{\circ}\right)$ joints at $\left|\bar{\phi}_{a R}\right|=7.33$ and $\left|\bar{\phi}_{a R}\right|=0.40$ respectively in Figures (6) and (7), it is noted that the stress field in Al-AS4 (0 ${ }^{\circ}$ ) lap joints is mostly due to thermal loading, whereas the stress field in Al-AS4 $\left(90^{\circ}\right)$ joint derives mostly from mechanical load. Therefore, the use of $\bar{\phi}_{a R}$ appears to be justified as a quick assessment of the relative importance of thermal and mechanical loads.

\section{Concluding remarks}

Two analytical models for the stress distribution in a symmetric double lap joint have been presented. The SO model assumes only shear stress exists in the adhesive, and produces a similar result to the work of Volkersen with the addition of thermal expansion. While not capturing peel stress or a traction free edge, it is a tractable solution with instructive non-dimensional parameters, and is therefore a useful tool for basic thermomechanical design of joints. The SP model, which is similar but more complex than the SO solution, does account for shear and peel stress. The 4 th order governing differential equation allows for proper representation of the traction free adhesive edge. Like the 


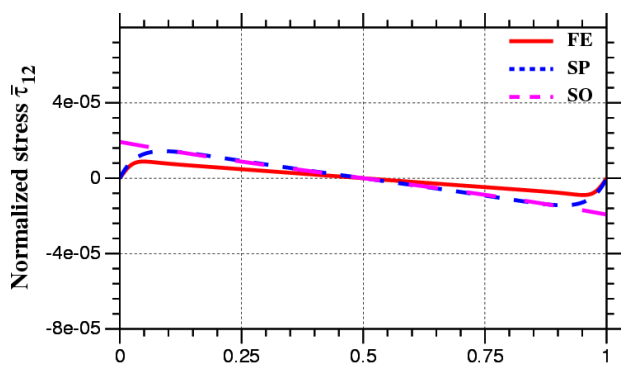

Normalized coordinate $\overline{\mathbf{x}}$

5.1: $\bar{\tau}_{b 12}$ due to $\bar{\phi}_{T}$

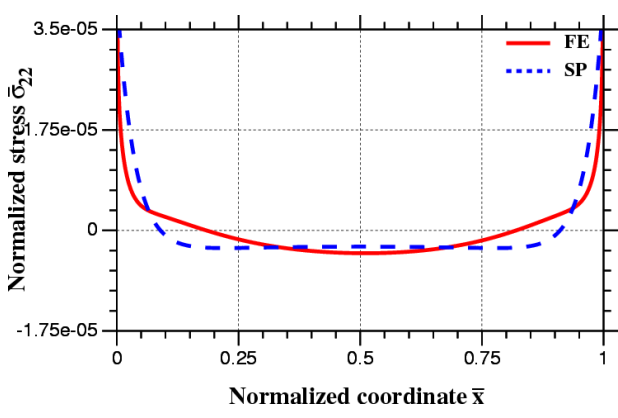

5.4: $\bar{\sigma}_{a 11}$ due to $\bar{\phi}_{T}$

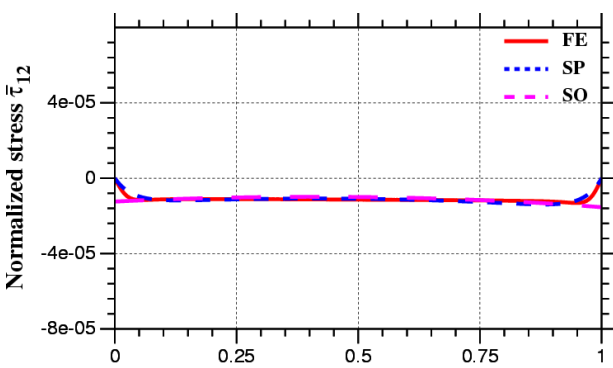

Normalized coordinate $\overline{\mathbf{x}}$

5.2: $\bar{\tau}_{b 12}$ due to $\bar{\phi}_{P}$

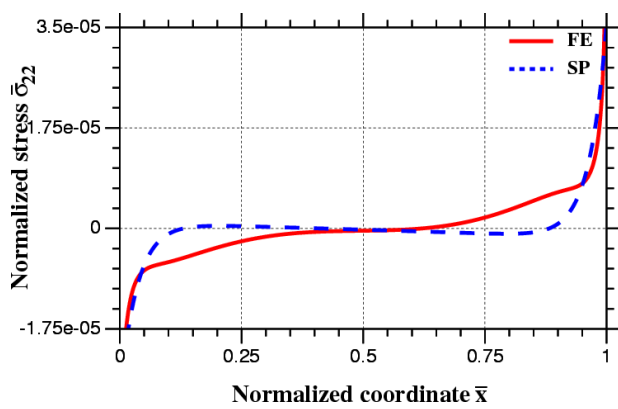

5.5: $\bar{\sigma}_{a 11}$ due to $\bar{\phi}_{P}$

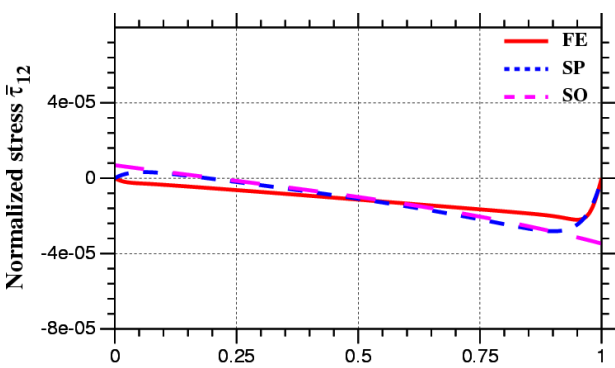

Normalized coordinate $\overline{\mathbf{x}}$

5.3: $\bar{\tau}_{b 12}$ due to $\bar{\phi}_{P}+\bar{\phi}_{T}$

Figure 5: FE, SP, and SO models of Al-Ti double lap joint with $1.0 \mathrm{~mm} \mathrm{FM300} \mathrm{adhesive.} \bar{\phi}_{a R}=-3.68$

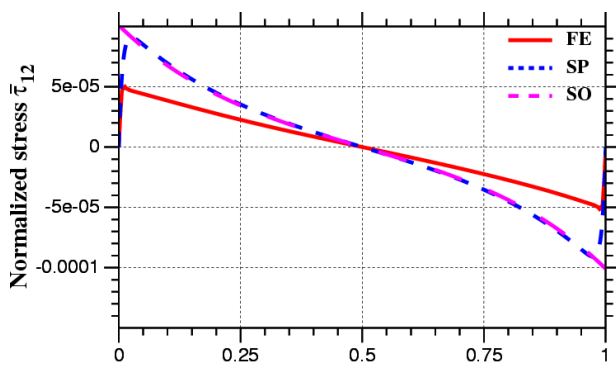

Normalized coordinate $\overline{\mathbf{x}}$

6.1: $\bar{\tau}_{b 12}$ due to $\bar{\phi}_{T}$

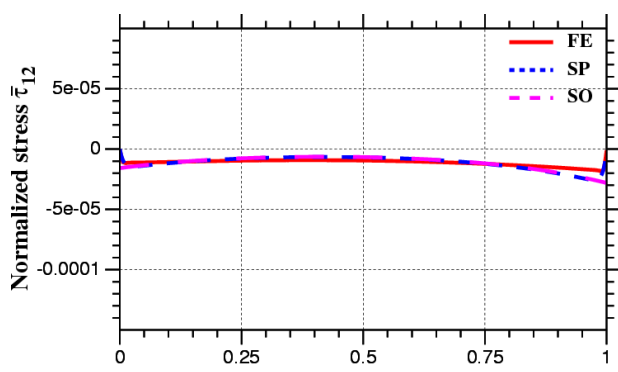

Normalized coordinate $\bar{x}$

6.2: $\bar{\tau}_{b 12}$ due to $\bar{\phi}_{P}$

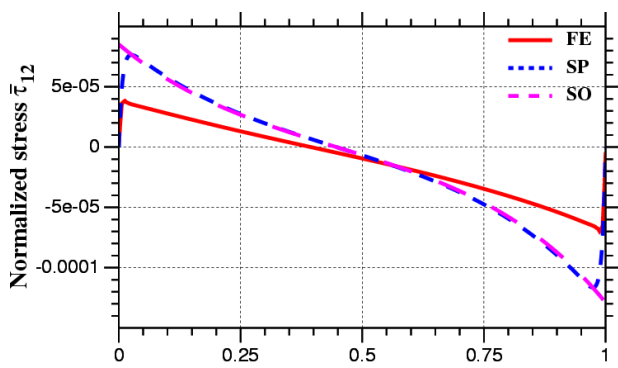

Normalized coordinate $\overline{\mathbf{x}}$

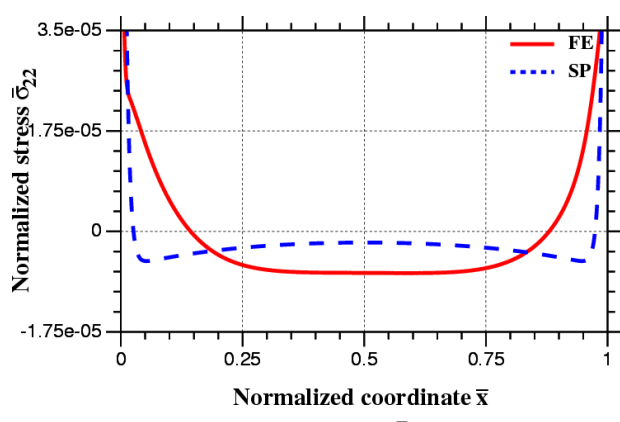

6.4: $\bar{\sigma}_{a 11}$ due to $\bar{\phi}_{T}$

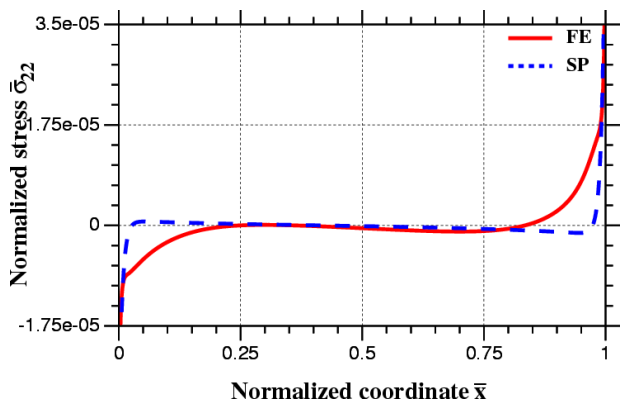

6.5: $\bar{\sigma}_{a 11}$ due to $\bar{\phi}_{P}$

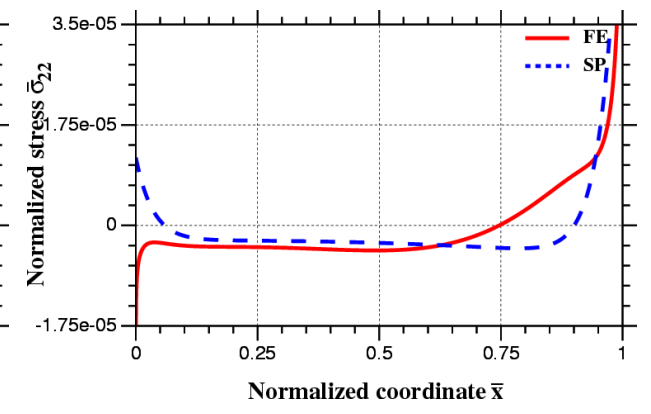

5.6: $\bar{\sigma}_{a 11}$ due to $\bar{\phi}_{P}+\bar{\phi}_{T}$ 


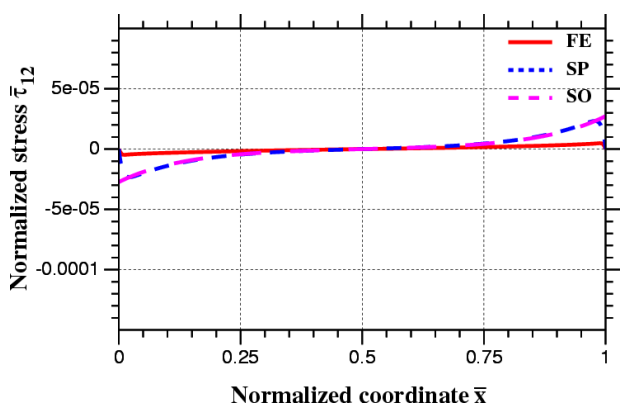

7.1: $\bar{\tau}_{b 12}$ due to $\bar{\phi}_{T}$
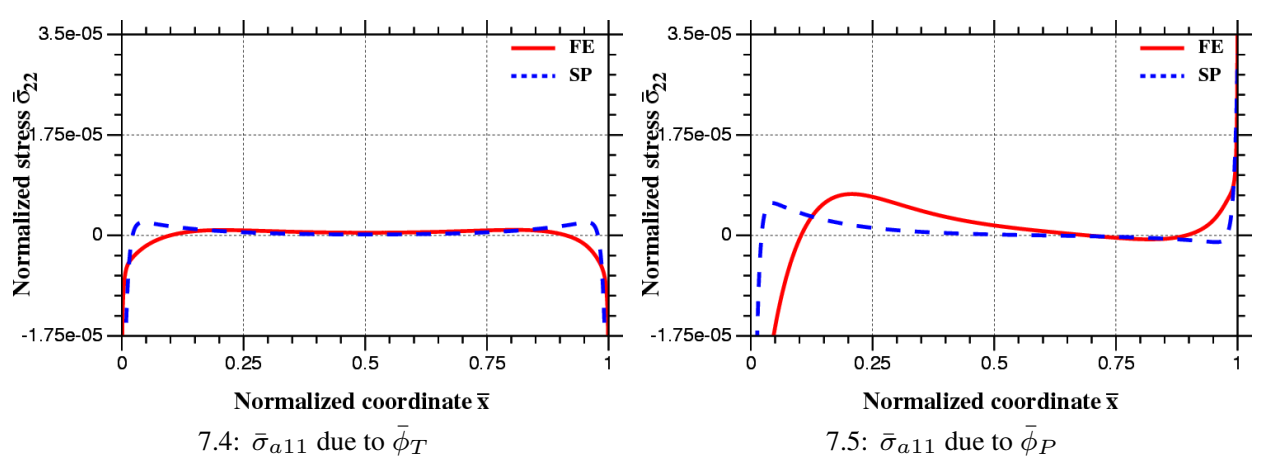

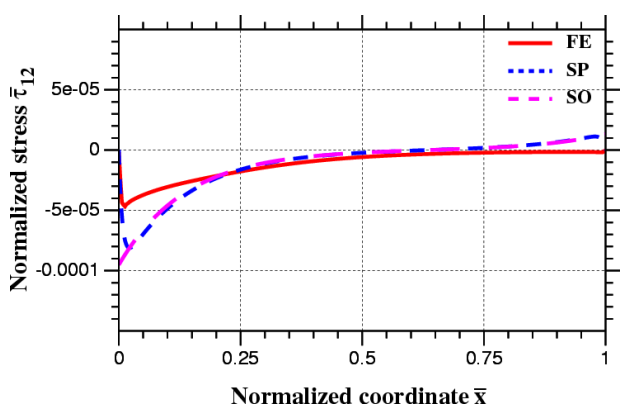

7.3: $\bar{\tau}_{b 12}$ due to $\bar{\phi}_{P}+\bar{\phi}_{T}$

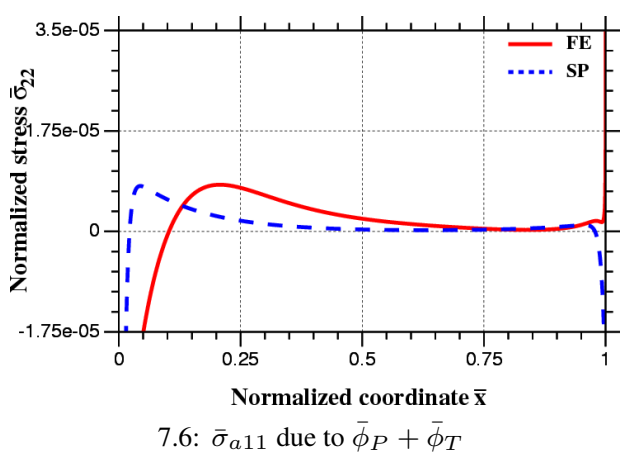

Figure 7: FE, SP, and SO models of Al-AS4 $\left(90^{\circ}\right)$ double lap joint with $0.2 \mathrm{~mm}$ FM300 adhesive. $\bar{\phi}_{a R}=0.40$

SO solution, the SP solution has instructive non-dimensional parameters which can be used as tools in joint design.

The SO and SP models both have good agreement in shear stress trend when compared with a FE model. The two solutions typically over predict the peak shear stresses, relative to the FE prediction. Peel stress predictions of the SP model are in good general agreement with the FE prediction, though caution must be used in interpreting the peel stress in both models, particularly when shear stresses from thermal and mechanical loads act against each other. Fortunately, this limitation arises in non-critical areas of the joint. For locations of peek peel stress in the joint, the FE and SP peel stress predictions are in good agreement.

In all joint combinations, there is only a small difference between results for SO and SP models. This difference is largest at the edges of the joint, where the SO solution does not capture the traction free condition. However, the SO model captures the majority of the stress field, and can be used as an initial design tool in situations where peel stress and the traction free boundary are not a concern. When peel stress becomes an important consideration, use of the SP model is required. If highly detailed stress predictions are required, the FE method is recommended.

A critical dimensionless parameter, $\bar{\phi}_{a R}$ (and its conjugate parameter $\bar{\phi}_{c R}$ ), has been identified which controls the stress distribution within the joint. It was shown that this ratio can be used as measure of the relative importance of mechanical and thermal loading in a joint of known (or expected) loading. This parameter can be identically derived using either the SO or SP solutions, making it a robust tool for quick assessments in joint design. The $\bar{\phi}_{a R}$ ratio also allows for isolation of the thermal and mechanical portions of the solution, which will facilitate an iterative solution when the combined thermal and mechanical loads are interdependent. 


\section{A. Boundary conditions for the SP solution}

The pre-simplified version of the longitudinal normal stress boundary conditions for the left and right edges respectively are:

$$
\begin{array}{r}
\bar{D}+\bar{C}+\bar{B}+\bar{A}-\frac{\bar{\phi}_{T}}{\bar{\gamma}}-\frac{\bar{\phi}_{P}}{\bar{\gamma}}=0, \\
e^{\bar{\lambda}_{4}} \bar{D}+e^{\bar{\lambda}_{2}} \bar{C}+e^{\bar{\lambda}_{2}} \bar{B}+e^{\bar{\lambda}_{1}} \bar{A}-\frac{\bar{\phi}_{T}}{\bar{\gamma}}-\frac{\bar{\phi}_{P}}{\bar{\gamma}}-\frac{2 P}{E_{a 11} t_{a}}=0 .
\end{array}
$$

When normalized by the total load $\bar{\phi}_{\text {total }}$, the normal stress boundary conditions become:

$$
\begin{array}{r}
\bar{D}+\bar{C}+\bar{B}+\bar{A}-\frac{1}{\bar{\gamma}}=0, \\
e^{\bar{\lambda}_{4}} \bar{D}+e^{\bar{\lambda}_{2}} \bar{C}+e^{\bar{\lambda}_{2}} \bar{B}+e^{\bar{\lambda}_{1}} \bar{A}-\frac{1}{\bar{\gamma}}-\frac{2 P}{E_{a 11} t_{a} \bar{\phi}_{\text {total }}}=0 .
\end{array}
$$

The pre-simplified version of the shear stress at the edges can be represented in either case by:

$$
\begin{aligned}
& \frac{3 \alpha_{b 33} E_{b 22} l^{4} \nu_{b 32} \Delta T}{E_{a 11} t_{a} t_{b} \nu_{b 23} \nu_{b 32}-E_{a 11} t_{a} t_{b}}+\frac{3 \alpha_{b 22} E_{b 22} l^{4} \Delta T}{E_{a 11} t_{a} t_{b} \nu_{b 23} \nu_{b 32}-E_{a 11} t_{a} t_{b}} \\
& +\frac{\left(E_{p^{0}} l^{2} \bar{\lambda}_{4}^{2} t_{p} \nu_{b 23} \nu_{b 32}-E_{p^{0}} l^{2} \bar{\lambda}_{4}^{2} t_{p}-E_{b 22} l^{3} \bar{\lambda}_{4}\right) \bar{D}}{E_{p^{0}} t_{p} \nu_{b 23} \nu_{b 32}-E_{p^{0}} t_{p}} \\
& +\frac{\left(E_{p^{0}} l^{2} \bar{\lambda}_{3}^{2} t_{p} \nu_{b 23} \nu_{b 32}-E_{p^{0}} l^{2} \bar{\lambda}_{3}^{2} t_{p}-E_{b 22} l^{3} \bar{\lambda}_{3}\right) \bar{C}}{E_{p^{0}} t_{p} \nu_{b 23} \nu_{b 32}-E_{p^{0}} t_{p}} \\
& +\frac{\left(E_{p^{0}} l^{2} \bar{\lambda}_{2}^{2} t_{p} \nu_{b 23} \nu_{b 32}-E_{p^{0}} l^{2} \bar{\lambda}_{2}^{2} t_{p}-E_{b 22} l^{3} \bar{\lambda}_{2}\right) \bar{B}}{E_{p^{0}} t_{p} \nu_{b 23} \nu_{b 32}-E_{p^{0}} t_{p}} \\
& +\frac{\left(E_{p^{0}} l^{2} \bar{\lambda}_{1}^{2} t_{p} \nu_{b 23} \nu_{b 32}-E_{p^{0}} l^{2} \bar{\lambda}_{1}^{2} t_{p}-E_{b 22} l^{3} \bar{\lambda}_{1}\right) \bar{A}}{E_{p^{0}} t_{p} \nu_{b 23} \nu_{b 32}-E_{p^{0}} t_{p}}=0, \\
& \frac{3 \alpha_{b 33} E_{b 22} l^{4} \nu_{b 32} \Delta T}{E_{a 11} t_{a} t_{b} \nu_{b 23} \nu_{b 32}-E_{a 11} t_{a} t_{b}}+\frac{3 \alpha_{b 22} E_{b 22} l^{4} \Delta T}{E_{a 11} t_{a} t_{b} \nu_{b 23} \nu_{b 32}-E_{a 11} t_{a} t_{b}} \\
& +\frac{\left(E_{p^{l}} l^{2} \bar{\lambda}_{4}^{2} e^{\bar{\lambda}_{4}} t_{p} \nu_{b 23} \nu_{b 32}-E_{p^{l}} l^{2} \bar{\lambda}_{4}^{2} e^{\bar{\lambda}_{4}} t_{p}-E_{b 22} l^{3} \bar{\lambda}_{4} e^{\bar{\lambda}_{4}}\right) \bar{D}}{E_{p^{l}} t_{p} \nu_{b 23} \nu_{b 32}-E_{p^{l}} t_{p}} \\
& +\frac{\left(E_{p^{l}} l^{2} \bar{\lambda}_{3}^{2} e^{\bar{\lambda}_{3}} t_{p} \nu_{b 23} \nu_{b 32}-E_{p^{l}} l^{2} \bar{\lambda}_{3}^{2} e^{\bar{\lambda}_{3}} t_{p}-E_{b 22} l^{3} \bar{\lambda}_{3} e^{\bar{\lambda}_{3}}\right) \bar{C}}{E_{p^{l}} t_{p} \nu_{b 23} \nu_{b 32}-E_{p^{l}} t_{p}} \\
& +\frac{\left(E_{p^{l}} l^{2} \bar{\lambda}_{2}^{2} e^{\bar{\lambda}_{2}} t_{p} \nu_{b 23} \nu_{b 32}-E_{p^{l}} l^{2} \bar{\lambda}_{2}^{2} e^{\bar{\lambda}_{2}} t_{p}-E_{b 22} l^{3} \bar{\lambda}_{2} e^{\bar{\lambda}_{2}}\right) \bar{B}}{E_{p^{l}} t_{p} \nu_{b 23} \nu_{b 32}-E_{p^{l}} t_{p}} \\
& +\frac{\left(E_{p^{l}} l^{2} \bar{\lambda}_{1}^{2} e^{\bar{\lambda}_{1}} t_{p} \nu_{b 23} \nu_{b 32}-E_{p^{l}} l^{2} \bar{\lambda}_{1}^{2} e^{\bar{\lambda}_{1}} t_{p}-E_{b 22} l^{3} \bar{\lambda}_{1} e^{\bar{\lambda}_{1}}\right) \bar{A}}{E_{p^{l}} t_{p} \nu_{b 23} \nu_{b 32}-E_{p^{l}} t_{p}}=0 .
\end{aligned}
$$

\section{B. Definition of the solution parameters}

\section{A. System parameters $\bar{\lambda}_{[12]}$ in terms of the orthotropic material properties}

$$
\bar{\lambda}_{[12]}^{2}=\frac{ \pm \sqrt{\frac{9 E_{b 22}^{2} l^{4}}{4 G b_{12}^{2} t_{b}^{4}\left(\nu_{b 23} \nu_{b 32}-1\right)^{2}}-\frac{12 E_{b 22} l^{4}\left(E_{a 11} t_{a} \nu_{c 13} \nu_{c 31}+2 E_{c 11} t_{c} \nu_{a 13} \nu_{a 31}-2 E_{c 11} t_{c}-E_{a 11} t_{a}\right)}{E_{a 11} E_{c 11} t_{a} t_{b}^{3} t_{c}\left(\nu_{b 23} \nu_{b 32}-1\right)}}-\frac{3 E_{b 22} l^{2}}{2 G b_{12} t_{b}^{2}\left(\nu_{b 23} \nu_{b 32}-1\right)}}{2}
$$




\section{B. $\quad \mu$ parameters for the SP solution integration constants.}

The $\mu$ values of Eqs. (27) and (37) are given by:

$$
\begin{aligned}
& \mu_{A_{T}}= \frac{\bar{\lambda}_{2}\left(e^{\bar{\lambda}_{2}}-1\right)}{\bar{\gamma}} \\
& \mu_{B_{T}}= \frac{e^{\bar{\lambda}_{1}} \bar{\lambda}_{2}\left(e^{\bar{\lambda}_{2}}-1\right)}{\bar{\gamma}} \\
& \mu_{C_{T}}=-\frac{\bar{\lambda}_{1}\left(e^{\bar{\lambda}_{1}}-1\right)}{\bar{\gamma}} \\
& \mu_{D_{T}}=-\frac{\bar{\lambda}_{1}\left(e^{\bar{\lambda}_{1}}-1\right) e^{\bar{\lambda}_{2}}}{\bar{\gamma}} \\
& \mu_{A_{P}}=-\left(\bar{\lambda}_{2} e^{2 \bar{\lambda}_{2}+\bar{\lambda}_{1}}-\bar{\lambda}_{1} e^{2 \bar{\lambda}_{2}+\bar{\lambda}_{1}}+2 \bar{\lambda}_{1} e^{\bar{\lambda}_{2}}-e^{\bar{\lambda}_{1}} \bar{\lambda}_{2}-\bar{\lambda}_{1} e^{\bar{\lambda}_{1}}\right) \\
& \mu_{B_{P}}=e^{\bar{\lambda}_{1}}\left(-2 \bar{\lambda}_{1} e^{\bar{\lambda}_{2}+\bar{\lambda}_{1}}+\bar{\lambda}_{2} e^{2 \bar{\lambda}_{2}}+\bar{\lambda}_{1} e^{2 \bar{\lambda}_{2}}-\bar{\lambda}_{2}+\bar{\lambda}_{1}\right) \\
& \mu_{C_{P}}= \frac{\bar{\lambda}_{1}\left(\bar{\lambda}_{2} e^{\bar{\lambda}_{2}+2 \bar{\lambda}_{1}}-\bar{\lambda}_{1} e^{\bar{\lambda}_{2}+2 \bar{\lambda}_{1}}+\bar{\lambda}_{2} e^{\bar{\lambda}_{2}}+\bar{\lambda}_{1} e^{\bar{\lambda}_{2}}-2 e^{\bar{\lambda}_{1}} \bar{\lambda}_{2}\right)}{\bar{\lambda}_{2}} \\
& \mu_{D_{P}}=-\frac{\bar{\lambda}_{1} e^{\bar{\lambda}_{2}}\left(2 \bar{\lambda}_{2} e^{\bar{\lambda}_{2}+\bar{\lambda}_{1}}-e^{2 \bar{\lambda}_{1}} \bar{\lambda}_{2}-\bar{\lambda}_{2}-\bar{\lambda}_{1} e^{2 \bar{\lambda}_{1}}+\bar{\lambda}_{1}\right)}{\bar{\lambda}_{2}} \\
& \mu_{1}= \bar{\lambda}_{2} e^{\bar{\lambda}_{2}+\bar{\lambda}_{1}}-\bar{\lambda}_{1} e^{\bar{\lambda}_{2}+\bar{\lambda}_{1}}+\bar{\lambda}_{2} e^{\bar{\lambda}_{2}}+\bar{\lambda}_{1} e^{\bar{\lambda}_{2}}-e^{\bar{\lambda}_{1}} \bar{\lambda}_{2}-\bar{\lambda}_{2}-\bar{\lambda}_{1} e^{\bar{\lambda}_{1}}+\bar{\lambda}_{1} \\
& \mu_{2}= \bar{\lambda}_{2} e^{\bar{\lambda}_{2}+\bar{\lambda}_{1}}-\bar{\lambda}_{1} e^{\bar{\lambda}_{2}+\bar{\lambda}_{1}}-\bar{\lambda}_{2} e^{\bar{\lambda}_{2}}-\bar{\lambda}_{1} e^{\bar{\lambda}_{2}}+e^{\bar{\lambda}_{1}} \bar{\lambda}_{2}-\bar{\lambda}_{2}+\bar{\lambda}_{1} e^{\bar{\lambda}_{1}}+\bar{\lambda}_{1} \\
& \mu_{3}= \frac{E_{c 11} \bar{\lambda}_{2} t_{b}^{3} t_{c}\left(\nu_{b 23} \nu_{b 32}-1\right)}{3 E_{b 22} l^{4}\left(\nu_{c 13} \nu_{c 31}-1\right)}
\end{aligned}
$$

\section{Acknowledgments}

This work was supported by the Space Vehicle Technology Institute under grant NCC3-989 jointly funded by NASA and DOD within the NASA Constellation University Institutes Project, with Claudia Meyer as the project manager. P.A. Gustafson acknowledges the work of Dr. Guido Dhondt and others on the CalculiX project, used as the FE solver for this work. P.A. Gustafson also extends gratitude to all contributors to the Maxima and Octave projects, which were used for symbolic and numerical manipulations respectively. Much of this work was completed using open source software licensed under the GNU General Public License (GPL) or one of its variants.

\section{References}

${ }^{1}$ Her, S.-C., "Stress analysis of adhesively-bonded lap joints," Composite Structures, Vol. 47, No. 1-4, Dec. 1999, pp. 673-678.

${ }^{2}$ Zhang, J., Collier, C., Bansal, Y., Bednarcykb, B. A., and Pindera, M.-J., "Analysis of Adhesively Bonded Composite Joints using a higherorder theory," 45th AIAA/ASME/ASCE/AHS/ASC Structures, Structrual Dynamics and Materials Conference, 2004.

${ }^{3}$ Volkersen, O., "Die Niektraftverteilung in Zugbeanspruchten mit Konstanten Laschenquerschritten," Luftfahrtforschung, Vol. 15, 1938, pp. 41-47.

${ }^{4}$ Goland, M. and Reissner, E., "The stresses in cemented joints," Journal of Applied Mechanics, Vol. 66, 1944.

${ }^{5}$ Hart-Smith, L., "Adhesive-Bonded Single-Lap Joints," NASA Contractor Report 112236, 1973.

${ }^{6}$ Peppiatt, N., Stress analysis of adhesive joints, Ph.D. thesis, University of Bristol, 1974.

${ }^{7}$ Renton, W. and Vinson, J., "The efficient design of adhesive bonded joints," Journal of .Adhesion, Vol. 7, 1975, pp. 175-193.

${ }^{8}$ Allman, D., "A Theory For Elastic Stresses In Adhesive Bonded Lap Joints," The Quarterly journal of mechanics and applied mathematics, Vol. 30, 1977, pp. 415.

${ }^{9}$ Yang, C., Huang, H., Tomblin, J. S., and Sun, W., "Elastic-plastic Model of Adhesive-bonded Single-lap Composite Joints," Journal of Composite Materials, Vol. 38, No. 4, 2004, pp. 293-309.

${ }^{10}$ Bensen, N., "Influence of stress distribution on the strength of bonded joints," Adhesion-Fundamentals and Practice, edited by N. Benson, MacLaren, September 1969, pp. 191-205, Adhesion: fundamentals and practice: a report of an international conference held at the University of Nottingham, England, 20-22 September 1966 by the Ministry of Technology (United Kingdom). ISBN 0853340250. 
${ }^{11}$ Adams, R., Comyn, J., and Wake, W., Structural Adhesive Joints in Engineering, Chapman and Hall, 1997.

${ }^{12}$ Hart-Smith, L., “Adhesive-Bonded Double-Lap Joints,” NASA Contractor Report 112235, 1973.

${ }^{13}$ Gilibert, Y. and Rigolot, A., "Determination of Stress Distribution in Double-Lap Joints, Matched Asymptotic Expansions and Conformal Mapping," Adhesively Bonded Joints: Testing, Analysis, and Design, edited by W. Johson, Vol. ASTM STP 981, American Society for Testing and Materials, Philadelphia, 1988, pp. 145-159.

${ }^{14}$ Sen, J. K. and Jones, R. M., "Stresses in double-lap joints bonded with a viscoelastic adhesive: Part I. Theory and experimental corroboration," AIAA Journal, Vol. 18, No. 10, October 1980, pp. 1237-1244.

${ }^{15}$ Sen, J. K. and Jones, R. M., "Stresses in double-lap joints bonded with a viscoelastic adhesive: Part II. Parametric study and joint design," AIAA Journal, Vol. 18, No. 11, November 1980, pp. 1376-1382.

${ }^{16}$ Chen, W. and Nelson, C., "Thermal Stress in Bonded Joints," IBM Journal of Research and Development, 1979, pp. 179-188.

${ }^{17}$ Whitney, J. M., "Stress analysis of a composite double-lap joint using higher order plate theory," AIAA, 1997, pp. 2004-2009.

${ }^{18}$ Kilic, B., Madenci, E., and Ambur, D., "Global-local finite element analysis of bonded single-lap joints," 45th AIAA/ASME/ASCE/AHS/ASC Structures, Structrual Dynamics and Materials Conference, 2004.

${ }^{19}$ Vinson, J. R. and Zumsteg, J. R., "Analysis of bonded joints in composite material structures including hygrothermal effects," Structures, Structural Dynamics, and Materials Conference, No. 20th, AIAA/ASME/ASCE/AHS, St. Louis, Mo, 1979, pp. 291-302.

${ }^{20}$ Adams, R. D., Coppendale, J., Mallick, V., and Al-Hamdan, H., "The effect of temperature on the strength of adhesive joints," International Journal of Adhesion and Adhesives, Vol. 12, No. 3, July 1992, pp. 185-190.

${ }^{21}$ Davies, G., Virtual work in structural analysis, John Wiley \& Sons, 1982.

${ }^{22}$ ASTM International, ASTM D 3528-96 Standard Test Method for Strength Properties of Double Lap Shear Adhesive Joints by Tension Loading.

${ }^{23}$ Dhondt, G., CalculiX CrunchiX user's manual version 1.5, 2006.

${ }^{24}$ Herakovich, C. T., Mechanics of fibrous composites, John Wiley and Sons, Inc, 1998. 